\title{
Probable Topiramate-Induced Hemiparesis
}

\author{
Joel Lamoure, Jessica Stovel, and Praful Chandarana
}

\section{INTRODUCTION}

$\mathrm{T}$ opiramate is a broad-spectrum antiepileptic medication that is used as monotherapy or in combination with other medications to treat epilepsy in both adults and children. ${ }^{1}$ It is also indicated for migraine prophylaxis in adults. ${ }^{1,2}$ An off-label use of topiramate is as a mood stabilizer (with no associated weight gain) in rapid-cycling and mixed bipolar episodes. ${ }^{3-7}$ The mechanisms of action of topiramate suggest a broad spectrum of anticonvulsant activity, including blockade of voltage-gated sodium and calcium channels, increase in inhibitory transmission via enhancement of $\gamma$-aminobutyric acid-stimulated chloride currents, decrease in excitatory transmission via blockade of the adenosine monophosphate-kainate subtype of the glutamate receptor, and weak inhibition of carbonic anhydrase..$^{8-11}$

Despite the benefits of topiramate as listed above, including weight neutrality, patients are still prone to discontinue their medications. This problem is highly prevalent among patients with mental health conditions, most notably schizophrenia and bipolar disorders. In particular, patients may abruptly discontinue their antiepileptic medications secondary to experiencing adverse effects, even if they have been made aware of the potential for such adverse effects through education provided by a pharmacist. Prescribers and other health care providers must be aware of potential adverse effects that may arise with the commencment and maintenance of antiepileptic therapy. ${ }^{12}$ Troublesome and relatively common adverse effects associated with topiramate that have been documented in the literature include paresthesia, fatigue, nausea, anorexia, anorgasmia, and changes in memory and concentration. Less common but serious adverse effects include hemiparesis, hypesthesia, abnormal coordination, chest pain, anemia, and bone marrow suppression. ${ }^{1,2,13-15}$ Many of these adverse effects are dose-related and result from too-rapid dose titration. ${ }^{16}$

As noted above, a common adverse effect of topiramate is paresthesia, which is characterized by abnormal neurologic sensations such as numbness, tingling, burning, prickling, and hypesthesia. In contrast, hemiparesis, defined as weakness of one side of the body, is much more serious. Although parasthesias are thought to be dose-related and occur in $51 \%$ of patients taking doses that exceed $100 \mathrm{mg} /$ day, ${ }^{12}$ there is no mention of hemiparesis in the product monograph, ${ }^{1}$ and only 3 cases of hemiparesis have been reported in the literature. ${ }^{9,17}$ The case reported here illustrates morbidity associated with probable topiramate-induced hemiparesis.

\section{CASE REPORT}

A 36-year-old woman* with diagnoses of epilepsy (with no seizures reported for 10 years), schizoaffective disorder, depression, and substance abuse presented to hospital with left-sided weakness, left-sided facial droop, slurred speech, decreased cognitive function, slowed motor skills, shuffling gait, recent history of falls, and flat affect. Of most concern to the patient were the left-sided weakness, acute dysphoria (abnormal depression and discontent), and slowed motor skills. Specifically, she had difficulty initiating movements and had "slowed down" considerably (e.g., had difficulty climbing stairs and reaching for items and was shuffling rather than walking). She also reported an increase in the intensity of headaches. She was admitted for investigation of possible stroke on February 20 (day 1). The symptoms had been present for 3 weeks before admission and had worsened acutely 3 days before admission.

Medication reconciliation at the time of admission, based on information provided by the patient, identified the following medication regimen: topiramate $100 \mathrm{mg}$ PO daily, zuclopenthixol $10 \mathrm{mg}$ PO bid, benztropine $2 \mathrm{mg}$ PO daily, clonazepam $0.5 \mathrm{mg}$ PO tid, lamotrigine $300 \mathrm{mg}$ PO qhs, and citalopram $60 \mathrm{mg}$ PO daily.

\footnotetext{
*To protect the privacy of the individual described here, all unique identifying information not pertinent to the case has been omitted from this report, in accord with standards of the Office of Research Ethics, University of Western Ontario.
} 
Recent changes to these medications were an increase in the dose of topiramate to $100 \mathrm{mg} 1 \mathrm{month}$ before admission; selfdecrease in the dose of zuclopenthixol, because of tremor, just before admission; and initiation of benztropine 1 week before admission.

The patient's vital signs on presentation were blood pressure $97 / 66 \mathrm{~mm} \mathrm{Hg}$, temperature $36.1^{\circ} \mathrm{C}$, pulse $63 / \mathrm{min}$, respiration rate $20 / \mathrm{min}$, and oxygen saturation $97 \%$. The findings of a physical examination included left-sided facial drooping, with full muscle tone on the right side. The neurologic exam yielded a rating of 4 out of 5 for muscle strength on the patient's left side, with no Babinski sign for the left foot. All laboratory values from samples drawn at admission were within normal limits. Finally, relevant imaging procedures completed at the time of admission included computed tomography of the head, which showed no significant intracranial abnormalities.

It was hypothesized that the left-sided effects and the hemiparesis were secondary to the use of topiramate, and tapering was proposed to wean the patient off this drug. On days 2 and 3, the patient received topiramate $75 \mathrm{mg}$ PO daily, and the dose was further reduced, to $50 \mathrm{mg}$ PO daily, on days 4 and 5 . On day 6, the topiramate was discontinued. Initially, there was some concern that the hemiparesis might also have been related to the zuclopenthixol, and tapering of that drug was also initiated. Because the patient had psychotic feautures and because there was a mood component to the presentation, quetiapine was initiated in hospital. The quetiapine was then tapered up as the zuclopenthixol was gradually tapered off. Tapering of zuclopenthixol took longer than tapering of topiramate, so the patient was still receiving zuclopenthixol when the topiramate tapering was complete. The hemiparesis symptoms resolved while the patient was still receiving zuclopenthixol. Once the zuclopenthixol was discontinued, it was determined that benztropine was no longer indicated, and this drug was abruptly discontinued. Given the patient's history of substance abuse, clonazepam was tapered off during the hospital stay, and zopiclone, on an as-needed basis, was initiated at a dose of $5 \mathrm{mg}$ hs prn. The daily dose of citalopram was reduced to $40 \mathrm{mg}$ on day 8 of the admission. The patient was steadier by day 5 of the admission, and all neurologic sequelae had resolved by day 8 . The remainder of the 27-day admission was uneventful, and therapy focused on stabilizing the patient's mood and reducing command hallucinations.

Discharge medications were lamotrigine $300 \mathrm{mg}$ PO qhs, citalopram $40 \mathrm{mg}$ PO daily, quetiapine $700 \mathrm{mg}$ PO qhs, and zopiclone $5 \mathrm{mg}$ PO qhs prn.

\section{DISCUSSION}

In the case reported here, hemiparesis had developed 3 weeks before admission, with significant worsening in the 3 days before admission. Given this time course of events, the pharma- cist and the physician hypothesized that the hemiparesis was associated with the use of topiramate and the recent increase in the dose of this drug. The patient's symptoms diminished markedly with a reduction in the dose of topiramate over 6 days and resolved completely with discontinuation of this medication. The Naranjo adverse reaction probability score ${ }^{18}$ was calculated to assess the probability that this adverse neurologic reaction was associated with topiramate. The score for this patient was 5 , which suggested a probable adverse drug reaction. There was no rechallenge with topiramate after discontinuation.

For the analysis of this case, it was of interest to determine whether topiramate-induced hemiparesis had been reported previously. A MEDLINE search (restricted to English-language articles) was performed for the period from year 1966 to November 2009, using the following Medical Subject Headings (MeSH terms): "topiramate", "hemiplegia", and "paresis". This search yielded published reports of 3 cases of hemiparesis, 2 in adult patients and 1 in a pediatric patient. Both adult patients had pre-existing compromise of neurologic function, whereas the pediatric patient did not, but all presented similarly to the patient described in the current report.

The first patient was a 41-year-old man with cerebral palsy (with resultant left hemispheric atrophy) and a history of daily complex partial seizures that were inadequately controlled by a number of anticonvulsants, including carbamazepine, sodium valproate, gabapentin, and diazepam. ${ }^{17}$ Following the initiation of topiramate, titrated to $25 \mathrm{mg}$ twice daily, the patient experienced fatigue, slurred speech, and left-sided weakness, which reduced his ability to bear weight. The topiramate was withdrawn, and the weakness gradually resolved by 8 weeks after discontinuation.

The second patient was a 59-year-old woman with generalized seizures secondary to herpes simplex encephalitis and resultant infarction of the left anterior temporal lobe. ${ }^{17}$ The seizures were poorly controlled with carbamazepine and phenytoin. Following the initiation of topiramate, which was increased over 2 months to a dose of $100 \mathrm{mg}$ twice daily, the patient experienced reduced tone and power in her right arm and leg. The topiramate was withdrawn, and the reduced tone and power resolved within 2 weeks after discontinuation.

The third report of topiramate-induced hemiparesis occurred in a pediatric patient. ${ }^{9}$ This 5-year-old child had a 3-year history of focal epilepsy characterized by facial twitching, inability to talk, and occasional short-lived postictal paresis of the right arm. Unlike the 2 adults described in the other report, the child had no pre-existing focal neurologic deficits. Despite therapy with phenytoin, carbamazepine, and valproic acid, the patient's seizures remained uncontrolled. Following introduction of topiramate, titrated over 6 months to $9 \mathrm{mg} / \mathrm{kg}$ daily (the high end of the recommended maintenance dose range), the 
parents noted that the child had difficulty chewing and swallowing and was experiencing reduced speech, deterioration of fine motor skills, and persistent weakness of the right arm. The topiramate was withdrawn, and the hemiparesis, difficulty finding words, and ataxia resolved within 1 month. ${ }^{9}$

In the case reported here, it was hypothesized that topiramate was responsible for the observed hemiparesis. Although the zuclopenthixol and topiramate were tapered at the same time, resolution of hemiparesis symptoms was noted while the patient was still receiving zuclopenthixol. Although the specific mechanism of this adverse reaction is unknown, one possible explanation for topiramate-induced hemiparesis in these patients is unmasking of a pre-existing compensated focal deficit and resultant seizures. ${ }^{9}$ Consequently, close clinical and electroencephalographic monitoring is essential for early detection of adverse reactions and prompt withdrawal of topiramate in such cases.

Topiramate is traditionally used as an anticonvulsant but has other indications and off-label uses, especially in psychiatry. Patients with mental health disorders often have medical comorbidities, multiple medications, adherence issues, and concurrent substance dependence. These patients may be more prone to adverse events secondary to their concurrent psychiatric and medical conditions. Thus, it is important that pharmacists monitor for symptoms such as hemiparesis, recognize these symptoms if they occur, and be able to respond appropriately, as well as proactively educating patients about such possible serious adverse effects. This case may help primary care pharmacists realize that topiramate may be a cause of hemiparesis and that withdrawal of this drug may be indicated for patients experiencing this adverse effect.

\section{References}

1. Topamax product monograph. Toronto (ON): Janssen-Ortho Inc.; 2009 Aug 19.

2. Brandes JL, Saper JR, Diamond M, Couch JR, Lewis DW, Schmitt J, et al. Topiramate for migraine prevention: a randomized controlled trial. JAMA 2004;291(8):965-973.

3. Yatham LN, Kennedy SH, O’Donovan C, Parikh SV, MacQueen G, McIntyre RS, et al.; Guidelines Group, CANMAT. Canadian Network for Mood and Anxiety Treatments (CANMAT) guidelines for the management of patients with bipolar disorder: update 2007. Bipolar Disord 2006; 8(6):721-739.

4. Ghaemi SN, Manwani SG, Katzow JJ, Ko JY, Goodwin FK. Topiramate treatment of bipolar spectrum disorders: a retrospective chart review. Ann Clin Psychiatry 2001;13(4):185-189.

5. Grunze HCR, Normann C, Langosch J, Schaefer M, Amann B, Sterr A, et al. Antimanic efficacy of topiramate in 11 patients in an open trial with an on-off-on design. J Clin Psychiatry 2001;62(6):464-468.
6. Marcotte D. Use of topiramate, a new anti-epileptic as a mood stabilizer. $J$ Affect Disord 1998;50(2-3):245-251.

7. McElroy SL, Suppes T, Keck PE, Frye MA, Denicoff KD, Altshuler LL, et al. Open-label adjunctive topiramate in the treatment of bipolar disorders. Biol Psychiatry 2000;47(12):1025-1033.

8. Dichter MA, Brodie MJ. New antiepileptic drugs. $N$ Engl J Med 1996;334(24):1583-90.

9. Patel H, Asconapé JJ, Garg BP. Reversible hemiparesis associated with the use of topiramate. Seizure 2002;11(7):460-463.

10. Kwan P, Sills GJ, Brodie MJ. The mechanisms of action of commonly used antiepileptic drugs. Pharmacol Ther 2001;90(1):21-34.

11. Deckers CL, Czuczwar SJ, Hekster YA, Keyser A, Kubova H, Meinardi H, et al. Selection of antiepileptic drug polytherapy based on mechanisms of action: the evidence reviewed. Epilepsia 2000;41(11):1364-1374.

12. Lamoure J, Stovel J. What are the troublesome side effects of topiramate? Can J Diagn 2007;24(12):25.

13. Elterman RD, Glauser TA, Wyllie E, Reife R, Wu SC, Pledger G; Topiramate YP Study Group. A double-blind, randomized trial of topiramate as adjunctive therapy for partial-onset seizures in children. Neurology 1999;52(7):1338-1344.

14. Sun C, Lay C, Broner S, Silberstein S, Tepper S, Newman L. Reversible anorgasmia with topiramate therapy for headache: a report of 7 patients. Headache 2006;46(9):1450-1453.

15. Brandes JL. Practical use of topiramate for migraine prevention. Headache 2005;45 Suppl 1:S66-S73.

16. Perucca E. A pharmacological and clinical review on topiramate, a new antiepileptic drug. Pharmacol Res 1997;35(4):241-256.

17. Stephen LJ, Maxwell JE, Brodie MJ. Transient hemiparesis with topiramate. BMJ 1999;318(7187):845.

18. Naranjo CA, Busto U, Sellers EM, Sandor P, Ruiz I, Roberts EA, et al. A method for estimating the probability of adverse drug reactions. Clin Pharmacol Ther 1981;30(2):239-45.

Joel Lamoure, RPh, BScPhm, FASCP, is with the Department of Pharmacy, London Health Sciences Centre, and the Department of Psychiatry, Schulich School of Medicine and Dentistry, University of Western Ontario, London, Ontario.

Jessica Stovel, RPh, HonBSc, BScPhm, ACPR, is with the Department of Pharmacy, London Health Sciences Centre, London, Ontario.

Praful Chandarana, MBChB, ABPN, FRCPC, is with the London Health Sciences Centre, and the Department of Psychiatry, Schulich School of Medicine and Dentistry, University of Western Ontario, London, Ontario.

\section{Address correspondence to:}

Joel Lamoure

Department of Psychiatry

London Health Sciences Centre

375 South Street, Office W707

London ON N6A 4 G5

e-mail: jlamour@uwo.ca 